\title{
Improving the reliability of the designed pipeline facilities by improving acoustic diagnostics of microdefects
}

\author{
Georgy Arshinov* \\ Kuban state agrarian University, 350044, Krasnodar, Russia
}

\begin{abstract}
The use of high-pressure pipelines for the transportation of oil products, natural gas, water requires ensuring the trouble-free operation of these structures during the operation period. The reliability and strength of pipelines determines their operational and economic reliability. Obviously, the presence of microdefects in pipelines material leads to a decrease in their strength. In the vicinity of microdefects, the process of material destruction is possible, leading to a loss of strength, which is accompanied by the destruction of structures and, as a consequence, economic damage, environmental deterioration. The study is aimed at developing the approaches and methods for increasing the economic and operational reliability of pipeline facilities by improving the acoustic methods for fixing microdefects in the used construction material. Improvement of acoustic methods for detecting microdefects in viscoelastic materials of pipelines can be carried out by developing mathematically refined models of the cylindrical shells' dynamics, taking into account real physical and mechanical characteristics, leading to more accurate parameters of combined nonlinear waves. Such models are nonlinear and are built taking into account the real hereditary properties of the material, the possibility of developing large strains in the material.
\end{abstract}

\section{Introduction}

Pipelines modeled by cylindrical shells are made of a material that may have inherited nonlinear physical and mechanical properties.

The trouble-free operation of pipelines under load depends on the material strength and determines the reliability of the structures. Improving the operational reliability of such structures is an urgent scientific problem.

One of the options for its solution is the improvement of non-destructive acoustic methods for diagnosing microdefects by mathematical modeling of the deformation waves appearance in cylindrical shells, which takes into account the viscoelastic physical and mechanical properties of the material and uses strict methods of mechanics heredity.

By experimentally measuring the velocity of a deformation wave in a cylindrical shell simulating a pipeline using nonlinear acoustic methods and comparing the measurement

\footnotetext{
* Corresponding author: arshinov_kts@mail.ru
} 
result with theoretically calculated values of velocities using the mathematical models that take into account the creep of materials, it is possible to locate microdefects in the vicinity of which the pipeline destruction under the influence of force loads can develop.

Therefore, the problem of determining more accurate values of the deformation wave velocity in a cylindrical shell using deformation models that take into account the creep properties of the material is urgent.

\section{Relevance of the research}

The economic and operational reliability of pipeline structures is highly dependent on their strength, which can be reduced due to the existence of microdefects in the structure. A decrease in strength can cause a loss of bearing capacity, destruction of a structure, accompanied by economic damage, environmental degradation.

Therefore, the improvement of acoustic search for microdefects in pipeline material using nonlinear mathematical models of deformation waves in cylindrical shells, taking into account their real physical and mechanical properties and determination of the specified wave parameters used in acoustic diagnostics, determine the relevance of the study.

\section{Methods}

The basis for increasing the reliability of pipelines is the material microdefects' acoustic detection improvement according to the refined wave characteristics obtained by mathematical modeling of nonlinear viscoelastic deformation waves in cylindrical shells.

The mathematical model of the wave process in a shell is constructed using rigorous methods of heredity mechanics, specifying the displacement fields of the medium points, Green tensor, the variational principle of mechanics, nonlinear properties of viscoelasticity and an asymptotic method for simplifying the equations describing deformation waves in the shell.

We will assume that the model of the pipeline is an infinite cylindrical shell. Let it have a thickness $h$ and a radius $\mathrm{R}$. We introduce a cylindrical coordinate system: we take the generatrix of the shell middle surface as the $\mathrm{x}$ axis, the tangent to the axial section as the $\mathrm{y}$ axis, and the normal to the middle surface of the cylindrical shell as the $\mathrm{z}$ axis. Let us suppose that external forces do not act on the shell (Fig. 1).

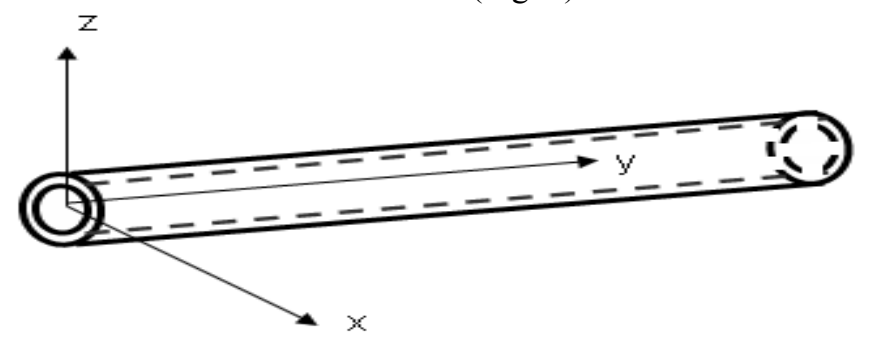

Fig. 1. Endless cylindrical shell

Using the Kirchhoff - Love hypothesis and neglecting the inertia of rotation leads to the following tensor of large deformations of the middle surface shell [1]:

$$
\varepsilon_{\mathrm{x}}^{\mathrm{z}}=\mathrm{U}_{\mathrm{x}}+\frac{1}{2}\left[\left(\mathrm{U}_{\mathrm{x}}-\mathrm{zW} \mathrm{xx}\right)^{2}+\left(\mathrm{V}_{\mathrm{x}}-\mathrm{zW}_{\mathrm{yy}}\right)^{2}+\mathrm{W}_{\mathrm{x}}^{2}\right]-\mathrm{zW}_{\mathrm{xx}}
$$




$$
\begin{aligned}
& \varepsilon_{\mathrm{y}}^{\mathrm{z}}=\mathrm{V}_{\mathrm{y}}-\mathrm{K}_{\mathrm{y}} \mathrm{W}+ \frac{1}{2}\left[\left(\mathrm{U}_{\mathrm{y}}-\mathrm{zW}_{\mathrm{xy}}\right)^{2}+\left(\mathrm{V}_{\mathrm{y}}-\mathrm{zW}_{\mathrm{yy}}\right)^{2}+\mathrm{W}_{\mathrm{y}}^{2}\right]-\mathrm{zW}_{\mathrm{yy}} \\
& \gamma^{\mathrm{z}}=\mathrm{U}_{\mathrm{y}}+\mathrm{V}_{\mathrm{x}}+\left(\mathrm{U}_{\mathrm{x}}-\mathrm{zW}_{\mathrm{xx}}\right)\left(\mathrm{U}_{\mathrm{y}}-\mathrm{zW}_{\mathrm{xy}}\right)+\left(\mathrm{V}_{\mathrm{x}}-\mathrm{zW}_{\mathrm{xy}}\right) \times \\
& \times\left(\mathrm{V}_{\mathrm{y}}-\mathrm{zW}_{\mathrm{yy}}\right)+\mathrm{W}_{\mathrm{x}} \mathrm{W}_{\mathrm{y}}-2 \mathrm{zW}_{\mathrm{xy}}
\end{aligned}
$$

where $\mathrm{U}, \mathrm{V}, \mathrm{W}$ - are the components of the points displacements vector along the axes $\mathrm{x}, \mathrm{y}, \mathrm{z}$. The variable $\mathrm{z}$ is the distance from the median surface to an arbitrary point in the shell layer, the value $\mathrm{K}_{\mathrm{y}}=\frac{1}{\mathrm{R}}$ specifies the shell curvature.

Let us set the physical and mechanical properties of the shell material by the equations of the linear theory of heredity, assuming volumetric deformations to be linearly elastic [2]:

$$
\begin{aligned}
& \sigma_{\mathrm{x}}^{\mathrm{z}}=\frac{\mathrm{E}}{1-v^{2}}\left(\varepsilon_{\mathrm{x}}+v \varepsilon_{\mathrm{y}}\right)-2 \mu \alpha \int_{-\infty}^{\mathrm{t}} \mathrm{e}^{-\beta(\mathrm{t}-\tau)} \mathrm{e}_{\mathrm{x}} \mathrm{d} \tau \\
& \sigma_{\mathrm{y}}^{\mathrm{z}}=\frac{\mathrm{E}}{1-v^{2}}\left(\varepsilon_{\mathrm{y}}+v \varepsilon_{\mathrm{x}}\right)-2 \mu \alpha \int_{-\infty}^{\mathrm{t}} \mathrm{e}^{-\beta(\mathrm{t}-\tau)} \mathrm{e}_{\mathrm{y}} \mathrm{d} \tau ;
\end{aligned}
$$

$$
\sigma_{x y}^{z}=\mu\left[\gamma-\alpha \int_{-\infty}^{t} e^{-\beta(t-\tau)} \gamma d \tau\right]
$$

in which $\mathrm{e}_{\mathrm{x}}=\varepsilon_{\mathrm{x}}-\frac{1}{3}\left(\varepsilon_{\mathrm{x}}+\varepsilon_{\mathrm{y}}\right) ; \mathrm{e}_{\mathrm{y}}=\varepsilon_{\mathrm{y}}-\frac{1}{3}\left(\varepsilon_{\mathrm{x}}+\varepsilon_{\mathrm{y}}\right)-$ are the components of the deformation deviator.

We use the expansion of the deformation components $\mathrm{e}_{\mathrm{x}} ; \mathrm{e}_{\mathrm{y}} ; \gamma$ in Taylor series by the degrees $(t-\tau)$, Let be $\beta \mathrm{t}>>1$, i.e., hereditary properties of the shell material quickly decay over time.

Leaving two terms in the expansion and dropping the superscript $\mathrm{z}$ in the Taylor series, we establish the following relationships between stresses and strains

$$
\begin{gathered}
\sigma_{x} \approx \frac{E}{1-v^{2}}\left(\varepsilon_{x}+v \varepsilon_{y}\right)+p\left[\frac{2}{3} \varepsilon_{x}-\frac{1}{3} \varepsilon_{y}\right] ; \\
\sigma_{y} \approx \frac{E}{1-v^{2}}\left(\varepsilon_{y}+v \varepsilon_{x}\right)+p\left[\frac{2}{3} \varepsilon_{y}-\frac{1}{3} \varepsilon_{x}\right] ; \\
\sigma_{x y}=\frac{p}{2} \gamma+\mu \gamma,
\end{gathered}
$$

in which the operator's action $p=2 \mu\left(\frac{\alpha}{\beta^{2}} \frac{\partial}{\partial t}-\frac{\alpha}{\beta}\right)$ per function $f(t)$ is given by the 
formula $\mathrm{pf}=2 \mu\left(\frac{\alpha}{\beta^{2}} \frac{\partial \mathrm{f}}{\partial \mathrm{t}}-\frac{\alpha}{\beta} \mathrm{f}\right)$.

Substituting the components of deformations (1) into (3), we determine the components of the stress tensor, expressed through the shell displacements:

$$
\begin{gathered}
\sigma_{\mathrm{x}}=\mathrm{N}\left[\mathrm{U}_{\mathrm{x}}+\frac{1}{2}\left(\mathrm{U}_{\mathrm{x}}^{2}-2 \mathrm{zU}_{\mathrm{x}} \mathrm{W}_{\mathrm{xx}}+\mathrm{z}^{2} \mathrm{~W}_{\mathrm{xx}}^{2}+\mathrm{V}_{\mathrm{x}}^{2}-2 \mathrm{zV}_{\mathrm{x}} \mathrm{W}_{\mathrm{xy}}+\mathrm{z}^{2} \mathrm{~W}_{\mathrm{xy}}^{2}+\mathrm{W}_{\mathrm{x}}^{2}\right)-\right. \\
\left.-\mathrm{zW}_{\mathrm{xx}}\right]+\mathrm{L}\left[\mathrm{V}_{\mathrm{y}}+\frac{1}{2}\left(\mathrm{U}_{\mathrm{y}}^{2}-2 \mathrm{zU}_{\mathrm{y}} \mathrm{W}_{\mathrm{xy}}+\mathrm{z}^{2} \mathrm{~W}_{\mathrm{xy}}^{2}+\mathrm{V}_{\mathrm{y}}^{2}-2 \mathrm{zV}_{\mathrm{y}} \mathrm{W}_{\mathrm{yy}}+\mathrm{z}^{2} \mathrm{~W}_{\mathrm{yy}}^{2}+\right.\right. \\
\left.\left.+\mathrm{W}_{\mathrm{y}}^{2}\right)-\mathrm{zW}_{\mathrm{yy}}\right] ; \\
\sigma_{\mathrm{y}}=\mathrm{N}\left[\mathrm{V}_{\mathrm{y}}-\mathrm{K}_{\mathrm{y}} \mathrm{W}+\frac{1}{2}\left(\mathrm{U}_{\mathrm{y}}^{2}-2 \mathrm{zU}_{\mathrm{y}} \mathrm{W}_{\mathrm{xy}}+\mathrm{z}^{2} \mathrm{~W}_{\mathrm{xy}}^{2}+\mathrm{V}_{\mathrm{y}}^{2}-2 \mathrm{zV}_{\mathrm{y}} \mathrm{W}_{\mathrm{yy}}+\mathrm{z}^{2} \mathrm{~W}_{\mathrm{yy}}^{2}+\right.\right. \\
\left.\left.+\mathrm{W}_{\mathrm{y}}^{2}\right)-\mathrm{zW} \mathrm{W}_{\mathrm{yy}}\right]+\mathrm{L}\left[\mathrm{U}_{\mathrm{x}}+\frac{1}{2}\left(\mathrm{U}_{\mathrm{x}}^{2}-2 \mathrm{zU}_{\mathrm{x}} \mathrm{W}_{\mathrm{xx}}+\mathrm{z}^{2} \mathrm{~W}_{\mathrm{xx}}^{2}+\mathrm{V}_{\mathrm{x}}^{2}-2 \mathrm{z} \mathrm{V}_{\mathrm{x}} \mathrm{W}_{\mathrm{xy}}+\right.\right. \\
\left.\left.\quad+\mathrm{z}^{2} \mathrm{~W}_{\mathrm{xy}}^{2}+\mathrm{W}_{\mathrm{x}}^{2}\right)-\mathrm{zW}_{\mathrm{xx}}\right] \\
\sigma_{\mathrm{xy}}=\mathrm{K}\left[\mathrm{U}_{\mathrm{y}}+\mathrm{V}_{\mathrm{x}}-2 \mathrm{zW} \mathrm{W}_{\mathrm{xy}}+\mathrm{U}_{\mathrm{x}} \mathrm{U}_{\mathrm{y}}-\mathrm{z}\left(\mathrm{U}_{\mathrm{x}} \mathrm{W}_{\mathrm{xy}}+\mathrm{U}_{\mathrm{y}} \mathrm{W}_{\mathrm{xx}}\right)+\mathrm{z}^{2} \mathrm{~W}_{\mathrm{xx}} \mathrm{W}_{\mathrm{xy}}+\mathrm{V}_{\mathrm{x}} \mathrm{V}_{\mathrm{y}}-\right. \\
\left.-\mathrm{z}\left(\mathrm{V}_{\mathrm{x}} \mathrm{W}_{\mathrm{yy}}+\mathrm{V}_{\mathrm{y}} \mathrm{W}_{\mathrm{xy}}\right)+\mathrm{z}^{2} \mathrm{~W}_{\mathrm{xy}} \mathrm{W}_{\mathrm{yy}}+\mathrm{W}_{\mathrm{x}} \mathrm{W}_{\mathrm{y}}\right]
\end{gathered}
$$

where

$$
\mathrm{N}=\frac{\mathrm{E}}{1-v^{2}}+\frac{2 \mathrm{p}}{3} ; \mathrm{L}=\frac{v \mathrm{E}}{1-v^{2}}-\frac{\mathrm{p}}{3} ; \mathrm{K}=\frac{\mathrm{E}}{2(1+v)}+\mathrm{p}
$$

Calculating the forces and moments in the shell element using the formulas [1]:

$$
\begin{aligned}
\mathrm{N}_{\mathrm{x}}=\int_{-\mathrm{h} / 2}^{\mathrm{h} / 2} \sigma_{\mathrm{x}} \mathrm{dz} ; & \mathrm{N}_{\mathrm{y}}=\int_{-\mathrm{h} / 2}^{\mathrm{h} / 2} \sigma_{\mathrm{y}} \mathrm{dz} ; & \mathrm{T}=\int_{-\mathrm{h} / 2}^{\mathrm{h} / 2} \tau \mathrm{dz} ; \\
\mathrm{M}_{\mathrm{x}}=\int_{-\mathrm{h} / 2}^{\mathrm{h} / 2} \sigma_{\mathrm{x}} \mathrm{zdz} ; & \mathrm{M}_{\mathrm{y}}=\int_{-\mathrm{h} / 2}^{\mathrm{h} / 2} \sigma_{\mathrm{y}} \mathrm{zdz} ; & \mathrm{H}=\int_{-\mathrm{h} / 2}^{\mathrm{h} / 2} \tau \mathrm{zdz} .
\end{aligned}
$$

we get the formulas

$$
\begin{aligned}
& \mathrm{N}_{\mathrm{x}}=\mathrm{N}\left\{\mathrm{hd}+\frac{\mathrm{h}^{3}}{24}\left(\mathrm{~W}_{\mathrm{xx}}^{2}+\mathrm{W}_{\mathrm{xy}}^{2}\right)\right\}+\mathrm{L}\left\{\mathrm{hb}+\frac{\mathrm{h}^{3}}{24}\left(\mathrm{~W}_{\mathrm{xy}}^{2}+\mathrm{W}_{\mathrm{yy}}^{2}\right)\right\} \\
& \mathrm{N}_{\mathrm{y}}=\mathrm{N}\left\{\mathrm{hb}+\frac{\mathrm{h}^{3}}{24}\left(\mathrm{~W}_{\mathrm{xy}}^{2}+\mathrm{W}_{\mathrm{yy}}^{2}\right)\right\}+\mathrm{L}\left\{\mathrm{hd}+\frac{\mathrm{h}^{3}}{24}\left(\mathrm{~W}_{\mathrm{xx}}^{2}+\mathrm{W}_{\mathrm{xy}}^{2}\right)\right\}
\end{aligned}
$$




$$
\begin{gathered}
\mathrm{T}=\mathrm{K}\left\{\mathrm{hc}+\frac{\mathrm{h}^{3}}{12}\left(\mathrm{~W}_{\mathrm{xx}} \mathrm{W}_{\mathrm{xy}}+\mathrm{W}_{\mathrm{xy}} \mathrm{W}_{\mathrm{yy}}\right)\right\} \\
\mathrm{M}_{\mathrm{x}}=-\frac{\mathrm{h}^{3}}{12}\{\mathrm{Nm}+\mathrm{Ln}\} ; \quad \mathrm{M}_{\mathrm{y}}=-\frac{\mathrm{h}^{3}}{12}\{\mathrm{Nn}+\mathrm{Lm}\} ; \quad \mathrm{H}=-\frac{\mathrm{h}^{3}}{12} \mathrm{Kl},
\end{gathered}
$$

where

$$
\begin{gathered}
\mathrm{d}=\mathrm{U}_{\mathrm{x}}+\frac{1}{2}\left(\mathrm{U}_{\mathrm{x}}^{2}+\mathrm{V}_{\mathrm{x}}^{2}+\mathrm{W}_{\mathrm{x}}^{2}\right) ; \mathrm{b}=\mathrm{V}_{\mathrm{y}}+\frac{1}{2}\left(\mathrm{U}_{\mathrm{y}}^{2}+\mathrm{V}_{\mathrm{y}}^{2}+\mathrm{W}_{\mathrm{y}}^{2}\right) ; \\
\mathrm{c}=\mathrm{U}_{\mathrm{y}}+\mathrm{V}_{\mathrm{x}}+\mathrm{U}_{\mathrm{x}} \mathrm{U}_{\mathrm{y}}+\mathrm{V}_{\mathrm{x}} \mathrm{V}_{\mathrm{y}}+\mathrm{W}_{\mathrm{x}} \mathrm{W}_{\mathrm{y}} ; \\
\mathrm{m}=2 \mathrm{U}_{\mathrm{x}} \mathrm{U}_{\mathrm{xx}}+2 \mathrm{~V}_{\mathrm{x}} \mathrm{W}_{\mathrm{xy}}+\mathrm{W}_{\mathrm{xx}} ; \mathrm{n}=2 \mathrm{U}_{\mathrm{y}} \mathrm{W}_{\mathrm{xy}}+2 \mathrm{~V}_{\mathrm{y}} \mathrm{W}_{\mathrm{yy}}+\mathrm{W}_{\mathrm{yy}} ; \\
\mathrm{q}=2 \mathrm{~W}_{\mathrm{xy}}+\mathrm{U}_{\mathrm{x}} \mathrm{W}_{\mathrm{xy}}+\mathrm{U}_{\mathrm{y}} \mathrm{W}_{\mathrm{xx}}+\mathrm{V}_{\mathrm{x}} \mathrm{W}_{\mathrm{yy}}+\mathrm{V}_{\mathrm{y}} \mathrm{W}_{\mathrm{xy}}
\end{gathered}
$$

We write down the shell motion equations [1]

$$
\begin{aligned}
& \frac{\partial \mathrm{N}_{\mathrm{x}}}{\partial \mathrm{x}}+\frac{\partial \mathrm{T}}{\partial \mathrm{y}}-\rho \mathrm{h} \frac{\partial^{2} \mathrm{U}}{\partial \mathrm{t}^{2}}=0 ; \frac{\partial \mathrm{T}}{\partial \mathrm{x}}+\frac{\partial \mathrm{N}_{\mathrm{y}}}{\partial \mathrm{y}}-\rho \mathrm{h} \frac{\partial^{2} \mathrm{~V}}{\partial \mathrm{t}^{2}}=0 ; \\
& \frac{\partial^{2} \mathrm{M}_{\mathrm{x}}}{\partial \mathrm{x}^{2}}+ \frac{\partial^{2} \mathrm{M}_{\mathrm{y}}}{\partial \mathrm{y}^{2}}+2 \frac{\partial^{2} \mathrm{H}}{\partial \mathrm{x} \partial \mathrm{y}}+\mathrm{K}_{\mathrm{y}} \mathrm{N}_{\mathrm{y}}+\frac{\partial}{\partial \mathrm{x}}\left(\mathrm{N}_{\mathrm{x}} \frac{\partial \mathrm{W}}{\partial \mathrm{x}}+\mathrm{T} \frac{\partial \mathrm{W}}{\partial \mathrm{y}}\right)+ \\
&+\frac{\partial}{\partial \mathrm{y}}\left(\mathrm{T} \frac{\partial \mathrm{W}}{\partial \mathrm{x}}+\mathrm{N}_{\mathrm{y}} \frac{\partial \mathrm{W}}{\partial \mathrm{y}}\right)-\rho \mathrm{h} \frac{\partial^{2} \mathrm{~W}}{\partial \mathrm{t}^{2}}=0
\end{aligned}
$$

in which we take into account the expressions for the efforts and moments, then the first equation of motion will take the form

$$
\begin{gathered}
\mathrm{N}\left\{\frac{\partial}{\partial \mathrm{x}}\left[\mathrm{U}_{\mathrm{x}}+\frac{1}{2}\left(\mathrm{U}_{\mathrm{x}}^{2}+\mathrm{V}_{\mathrm{x}}^{2}+\mathrm{W}_{\mathrm{x}}^{2}\right)+\frac{\mathrm{h}^{2}}{24}\left(\mathrm{~W}_{\mathrm{xx}}^{2}+\mathrm{W}_{\mathrm{xy}}^{2}\right)\right]\right\}+ \\
+\mathrm{L}\left\{\frac{\partial}{\partial \mathrm{x}}\left[\mathrm{V}_{\mathrm{y}}+\frac{1}{2}\left(\mathrm{U}_{\mathrm{y}}^{2}+\mathrm{V}_{\mathrm{y}}^{2}+\mathrm{W}_{\mathrm{y}}^{2}\right)+\frac{\mathrm{h}^{2}}{24}\left(\mathrm{~W}_{\mathrm{xy}}^{2}+\mathrm{W}_{\mathrm{yy}}^{2}\right)\right]\right\}+ \\
\left.\left.+\frac{\mathrm{h}^{2}}{12}\left(\mathrm{~W}_{\mathrm{xx}} \mathrm{W}_{\mathrm{xy}}+\mathrm{W}_{\mathrm{xy}} \mathrm{W}_{\mathrm{yy}}\right)\right]\right\}-\rho \frac{\partial^{2} \mathrm{U}}{\partial \mathrm{t}^{2}}=0 ;
\end{gathered}
$$

and we obtain the second from the first by replacing $\mathrm{U}$ for $\mathrm{V}, \mathrm{X}$ for $\mathrm{y}, \mathrm{V}$ for $\mathrm{U}, \mathrm{y}$ for $\mathrm{X}$.

The third equation of the shell motion in displacements takes the form:

$$
\frac{\partial^{2}}{\partial x^{2}}\left\{-\frac{h^{2}}{12}\left[N\left(2 U_{x} U_{x x}+2 V_{x} W_{x y}+W_{x x}\right)+L\left(2 U_{y} W_{x y}+2 V_{y} W_{y y}+W_{y y}\right)\right]\right\}+
$$




$$
\begin{aligned}
+\frac{\partial^{2}}{\partial \mathrm{y}^{2}}\left\{-\frac{\mathrm{h}^{3}}{12}\left[\mathrm{~N}\left(2 \mathrm{U}_{\mathrm{y}} \mathrm{W}_{\mathrm{xy}}+2 \mathrm{~V}_{\mathrm{y}} \mathrm{W}_{\mathrm{yy}}+\mathrm{W}_{\mathrm{yy}}\right)+\mathrm{L}\left(2 \mathrm{U}_{\mathrm{x}} \mathrm{U}_{\mathrm{xx}}+2 \mathrm{~V}_{\mathrm{x}} \mathrm{W}_{\mathrm{xy}}+\mathrm{W}_{\mathrm{xx}}\right)\right]\right\}+ \\
+2 \frac{\partial^{2}}{\partial \mathrm{x} \partial \mathrm{y}}\left\{-\frac{\mathrm{h}^{2}}{12} \mathrm{~K}\left(2 \mathrm{~W}_{\mathrm{xy}}+\mathrm{U}_{\mathrm{x}} \mathrm{W}_{\mathrm{xy}}+\mathrm{U}_{\mathrm{y}} \mathrm{W}_{\mathrm{xx}}+\mathrm{V}_{\mathrm{x}} \mathrm{W}_{\mathrm{yy}}+\mathrm{V}_{\mathrm{y}} \mathrm{W}_{\mathrm{xy}}\right)\right\}+ \\
+\frac{1}{\mathrm{R}}\left\{\mathrm{N}\left[\mathrm{V}_{\mathrm{y}}+\frac{1}{2}\left(\mathrm{U}_{\mathrm{y}}^{2}+\mathrm{V}_{\mathrm{y}}^{2}+\mathrm{W}_{\mathrm{y}}^{2}\right)+\frac{\mathrm{h}^{3}}{24}\left(\mathrm{~W}_{\mathrm{xy}}^{2}+\mathrm{W}_{\mathrm{yy}}^{2}\right)\right]+\right. \\
\left.+\mathrm{L}\left[\mathrm{U}_{\mathrm{x}}+\frac{1}{2}\left(\mathrm{U}_{\mathrm{x}}^{2}+\mathrm{V}_{\mathrm{x}}^{2}+\mathrm{W}_{\mathrm{x}}^{2}\right)+\frac{\mathrm{h}^{3}}{24}\left(\mathrm{~W}_{\mathrm{xx}}^{2}+\mathrm{W}_{\mathrm{xy}}^{2}\right)\right]\right\}=0 .
\end{aligned}
$$

Let us simplify the equations of motion, neglecting the terms in them higher than the second order of smallness, we obtain:

$$
\begin{gathered}
\frac{\partial}{\partial \mathrm{x}}\left\{\mathrm{N}\left[\mathrm{d}+\frac{\mathrm{h}^{2}}{24}\left(\mathrm{~W}_{\mathrm{xx}}^{2}+\mathrm{W}_{\mathrm{xy}}^{2}\right)\right]+\mathrm{L}\left[\mathrm{b}+\frac{\mathrm{h}^{2}}{24}\left(\mathrm{~W}_{\mathrm{xy}}^{2}+\mathrm{W}_{\mathrm{yy}}^{2}\right)\right]\right\}+ \\
+\frac{\partial}{\partial \mathrm{y}}\left\{\mathrm{K}\left[\mathrm{c}+\frac{\mathrm{h}^{2}}{12}\left(\mathrm{~W}_{\mathrm{xx}} \mathrm{W}_{\mathrm{xy}}+\mathrm{W}_{\mathrm{xy}} \mathrm{W}_{\mathrm{yy}}\right)\right]\right\}-\rho \frac{\partial^{2} \mathrm{U}}{\partial \mathrm{t}^{2}}=0 \\
\frac{\partial}{\partial \mathrm{x}}\left\{\mathrm{K}\left[\mathrm{c}+\frac{\mathrm{h}^{2}}{12}\left(\mathrm{~W}_{\mathrm{xx}} \mathrm{W}_{\mathrm{xy}}+\mathrm{W}_{\mathrm{xy}} \mathrm{W}_{\mathrm{yy}}\right)\right]\right\}+\frac{\partial}{\partial \mathrm{y}}\{\mathrm{N}[\mathrm{b}+ \\
\left.\left.+\frac{\mathrm{h}^{2}}{24}\left(\mathrm{~W}_{\mathrm{xy}}^{2}+\mathrm{W}_{\mathrm{yy}}^{2}\right)\right]+\mathrm{L}\left[\mathrm{d}+\frac{\mathrm{h}^{2}}{24}\left(\mathrm{~W}_{\mathrm{xx}}^{2}+\mathrm{W}_{\mathrm{xy}}^{2}\right)\right]\right\}-\rho \frac{\partial^{2} \mathrm{~V}}{\partial \mathrm{t}^{2}}=0 \\
\frac{\partial^{2}}{\partial \mathrm{x}^{2}}\left\{-\frac{\mathrm{h}^{2}}{12}(\mathrm{Nm}+\mathrm{Ln})\right\}+\frac{\partial^{2}}{\partial \mathrm{y}^{2}}\left\{-\frac{\mathrm{h}^{3}}{12}(\mathrm{Nn}+\mathrm{Lm})\right\}+ \\
+2 \frac{\partial^{2}}{\partial \mathrm{x} \partial \mathrm{y}}\left\{-\frac{\mathrm{h}^{2}}{12} \mathrm{Kq}\right\}+\frac{1}{\mathrm{R}}\left\{\mathrm{N}\left[\mathrm{b}+\frac{\mathrm{h}^{2}}{24}\left(\mathrm{~W}_{\mathrm{xy}}^{2}+\mathrm{W}_{\mathrm{yy}}^{2}\right)\right]+\mathrm{L}[\mathrm{d}+\right. \\
\left.\left.+\frac{\mathrm{h}^{2}}{24}\left(\mathrm{~W}_{\mathrm{xx}}^{2}+\mathrm{W}_{\mathrm{xy}}^{2}\right)\right]\right\}+\frac{\partial}{\partial \mathrm{x}}\left\{\left[\mathrm{N}\left[\mathrm{d}+\frac{\mathrm{h}^{2}}{24}\left(\mathrm{~W}_{\mathrm{xx}}^{2}+\mathrm{W}_{\mathrm{xy}}^{2}\right)\right]+\mathrm{L}[\mathrm{b}+\right.\right. \\
\left.\left.\left.+\frac{\mathrm{h}^{2}}{24}\left(\mathrm{~W}_{\mathrm{xy}}^{2}+\mathrm{W}_{\mathrm{yy}}^{2}\right)\right]\right] \mathrm{W}_{\mathrm{x}}+\left[\mathrm{K}\left[\mathrm{c}+\frac{\mathrm{h}^{2}}{12}\left(\mathrm{~W}_{\mathrm{xx}} \mathrm{W}_{\mathrm{xy}}+\mathrm{W}_{\mathrm{xy}} \mathrm{W} \mathrm{yy}\right)\right]\right] \mathrm{W}_{\mathrm{y}}\right\}+ \\
+\frac{\partial}{\partial \mathrm{y}}\left\{\left[\mathrm{K}\left[\mathrm{c}+\frac{\mathrm{h}^{2}}{12}\left(\mathrm{~W} \mathrm{~W}_{\mathrm{xx}} \mathrm{W}_{\mathrm{xy}}+\mathrm{W}_{\mathrm{xy}} \mathrm{W}_{\mathrm{yy}}\right)\right]\right] \mathrm{W}_{\mathrm{x}}+\right. \\
\left.+\left[\mathrm{N}\left[\mathrm{b}+\frac{\mathrm{h}^{2}}{24}\left(\mathrm{~W}_{\mathrm{xy}}^{2}+\mathrm{W}_{\mathrm{yy}}^{2}\right)\right]+\mathrm{L}\left[\mathrm{d}+\frac{\mathrm{h}^{2}}{24}\left(\mathrm{~W}_{\mathrm{xx}}^{2}+\mathrm{W}_{\mathrm{xy}}^{2}\right)\right]\right]\right\}-\rho \frac{\partial^{2} \mathrm{~W}}{\partial \mathrm{t}^{2}}=0
\end{gathered}
$$

The system of nonlinear (4) - (6) describes longitudinal deformation waves in a geometrically nonlinear viscoelastic cylindrical shell. 
Let us simplify the system by asymptotic methods, transforming the relations (4) - (6) to the dimensionless parameters

$$
\mathrm{U}=\mathrm{AU} * ; \mathrm{V}=\mathrm{AV} * ; \mathrm{W}=\mathrm{hW} * ; \quad \mathrm{x}=\mathrm{Lx} * ; \mathrm{y}=\mathrm{Ry} *
$$

where $\mathrm{A}, \mathrm{L}$ - are the strain amplitude and wavelength, $\mathrm{R}$ - is the shell radius.

Let us investigate long waves with small amplitude, assuming the following quantities to be small

$$
\varepsilon=\frac{\mathrm{A}}{\mathrm{L}} ; \delta_{1}=\frac{\sqrt{\mathrm{hR}}}{\mathrm{L}} ; \quad \delta_{2}=\frac{\mathrm{h}}{\mathrm{R}} ; \quad \delta_{3}=\frac{\mathrm{A}}{\mathrm{R}}
$$

This corresponds to the smallness of the shell thickness $\mathrm{h}$ compared with $\mathrm{R}$.

Let the parameters $\delta_{1}, \delta_{2}$ - be equivalent $\varepsilon$, then $\delta_{3}$ equivalent to $\sqrt{\varepsilon}$. We make the following change of variables

$$
\xi=x^{*}-\frac{\mathrm{c}_{1}}{\mathrm{~L}} \mathrm{t} ; \quad \eta=\varepsilon \mathrm{y}^{*} ; \quad \tau=\varepsilon \frac{\mathrm{c}_{1}}{\mathrm{~L}} \mathrm{t}
$$

Where $c_{1}-$ is an unknown quantity.

We use the asymptotic expansions of the functions $\mathrm{U}^{*}, \mathrm{~V}^{*}, \mathrm{~W}^{*}$, dropping asterisks under the variables:

$$
\mathrm{U}=\mathrm{U}_{0}+\varepsilon \mathrm{U}_{1}+\ldots ; \quad \mathrm{V}=\sqrt{\varepsilon}\left(\mathrm{V}_{0}+\varepsilon \mathrm{V}_{1}+\ldots,\right) ; \quad \mathrm{W}=\mathrm{W}_{0}+\varepsilon \mathrm{W}_{1}+\ldots
$$

We will consider the parameter $\frac{\alpha c_{1}}{\beta^{2} \mathrm{~L}}$ in the equations of motion equivalent $\varepsilon$, then from the zeroth approximation the system follows:

$$
\begin{gathered}
\mathrm{E}\left(\frac{\alpha_{1}}{6}-v\right) \frac{\mathrm{h}}{\mathrm{R} \varepsilon} \mathrm{W}_{0 \xi}=0 \\
{\left[\frac{1}{2} \mathrm{E}\left(1-v-\alpha_{1}\right)-\rho\left(1-v^{2}\right) \mathrm{c}_{1}^{2}\right] \mathrm{V}_{0 \xi \xi}+\mathrm{E}\left[\frac{\mathrm{A}}{\sqrt{\varepsilon \mathrm{R}}}\left(\frac{1+\mathrm{v}}{2}-\frac{\alpha_{1}}{3}\right) \mathrm{U}_{0 \xi \eta}+\right.} \\
\left.+\frac{\mathrm{hL}}{\mathrm{R}^{2} \sqrt{\varepsilon}}\left(\frac{\alpha_{1}}{3}-1\right) \mathrm{W}_{0 \eta}\right]=0 \\
\frac{\mathrm{h}}{\mathrm{R} \varepsilon} \mathrm{W}_{0}=v_{1} \mathrm{U}_{0 \xi}
\end{gathered}
$$

where

$$
\alpha_{1}=\frac{\alpha}{\beta(1+v)} ; v_{1}=\frac{3}{2} \frac{2 v-\alpha_{1}}{3-\alpha_{1}}
$$


Taking into account formula (9), from the equation (7) we obtain an expression for the deformation wave velocity

$$
c_{1}=\sqrt{\frac{E}{\rho} \frac{\alpha_{2}}{\left(1-v^{2}\right)}},
$$

where

$$
\alpha_{2}=1-\frac{\alpha_{1}}{3}+\frac{3}{2}\left(\frac{\alpha_{1}}{6}-v\right) \frac{2 v-\alpha_{1}}{3-\alpha_{1}}
$$

Speed $\mathrm{c}_{1}$ is valid for $\alpha_{2}>0$, which corresponds to the inequality $\alpha_{1}^{2}-24(1-v) \alpha_{1}+36\left(1-v^{2}\right)>0$. It will be fulfilled provided $\alpha_{1}<12(1-v)-6 \sqrt{(1-v)(3-5 v)}$ or $\quad \alpha_{1}>12(1-v)+6 \sqrt{(1-v)(3-5 v)}$, what can be achieved with a certain choice of physical parameters $\alpha, \beta, v$.

From the first approximation we obtain the following equations:

$$
\begin{gathered}
v_{1}\left(v-\frac{\alpha_{1}}{6}\right) \mathrm{U}_{1 \xi \xi}-\frac{\mathrm{h}}{\mathrm{R} \varepsilon}\left(v-\frac{\alpha_{1}}{6}\right) \mathrm{W}_{1 \xi \xi}+\frac{\mathrm{LA}}{2 \mathrm{R}^{2}}\left(1-v-\frac{\alpha_{1}}{2}\right) \mathrm{U}_{0 \eta \eta}+\frac{\mathrm{A}}{2 \mathrm{R} \sqrt{\varepsilon}}(1+v- \\
\left.-\frac{\alpha_{1}}{3}\right) \mathrm{V}_{0 \xi \eta}+\left(1-\frac{\alpha_{1}}{3}\right) \mathrm{U}_{0 \xi} \mathrm{U}_{0 \xi \xi}+\frac{\alpha_{1} \mathrm{c}_{1}}{2 \beta \mathrm{L} \varepsilon}\left(\frac{2}{3}-\frac{\mathrm{h} v_{1}}{3 \mathrm{R} \varepsilon}\right) \mathrm{U}_{0 \xi \xi \xi}+ \\
+\frac{2 \rho\left(1-v^{2}\right) \mathrm{c}_{1}^{2}}{\mathrm{E}} \mathrm{U}_{0 \xi \tau}=0
\end{gathered}
$$

$$
\begin{gathered}
\frac{\mathrm{A} l}{\mathrm{R}^{2}}\left(1-\frac{\alpha_{1}}{3}\right) \mathrm{V}_{0 \eta \eta}+\frac{1}{2}\left(1-v-\alpha_{1}-\alpha_{2}\right) \mathrm{V}_{1 \xi \xi}+\frac{\mathrm{A}}{\sqrt{\varepsilon} \mathrm{R}}\left(\frac{1+\mathrm{v}}{2}-\frac{\alpha_{1}}{3}\right) \mathrm{U}_{1 \xi \eta}+ \\
+\frac{\mathrm{hl}}{\mathrm{R}^{2} \sqrt{\varepsilon}}\left(\frac{\alpha_{1}}{3}-1\right) \mathrm{W}_{1 \eta}+\frac{\alpha_{1} \mathrm{c}_{1}}{2 \beta \varepsilon \mathrm{L}}\left(\mathrm{V}_{0 \xi \xi \xi}+\frac{2 \mathrm{~A}}{3 \mathrm{R} \sqrt{\varepsilon}} \mathrm{U}_{0 \xi \xi \eta}-\frac{2}{3} \frac{\mathrm{hl}}{\mathrm{R}^{2} \sqrt{\varepsilon}} \mathrm{W}_{0 \xi \eta}\right)- \\
-\frac{\rho\left(1-v^{2}\right)}{\mathrm{E}} \mathrm{V}_{1 \xi \xi}+\frac{2 \rho\left(1-v^{2}\right)}{\mathrm{E}} \mathrm{V}_{0 \xi \tau}=0 ; \\
\left(\frac{\mathrm{v}}{2}-\frac{\alpha_{1}}{12}\right) \mathrm{U}_{0 \xi}^{2}+\frac{\rho\left(1-v^{2}\right) \mathrm{c}_{1}^{2} \mathrm{Rh}}{\mathrm{VL}_{1} \mathrm{U}_{1 \xi}-\frac{\mathrm{h}}{\mathrm{R} \varepsilon} \mathrm{W}_{1}+\left(1+\frac{2}{3} \frac{\mathrm{A}}{\mathrm{R} \sqrt{\varepsilon}} \frac{\alpha_{1}}{2}\right) \mathrm{V}_{0 \eta}+}+\frac{\alpha_{1} \mathrm{c}_{1}}{2 \beta \varepsilon \mathrm{L}}\left(\frac{2}{3} \frac{\mathrm{h}}{\mathrm{R} \varepsilon} \mathrm{W}_{0 \xi}+\right. \\
\left.\frac{1}{3} \mathrm{U}_{0 \xi \xi}\right)=0 .
\end{gathered}
$$

Multiplying the equation (11) by $\left(v-\frac{\alpha_{1}}{6}\right)$, then differentiating it by $\xi$ and taking 
into account that $\mathrm{V}_{0 \xi}=\frac{\mathrm{U}_{0 \eta}}{\mathrm{A}_{2}}$, where

$$
\mathrm{A}_{2}=\frac{\alpha_{2}-\frac{1}{2}\left(1-v-\alpha_{1}\right)}{\frac{\mathrm{A}}{\sqrt{\varepsilon} \mathrm{R}}\left(\frac{1+v}{2}-\frac{\alpha_{1}}{3}\right)+\frac{1 \sqrt{\varepsilon} v_{1}}{\mathrm{R}}\left(\frac{\alpha_{1}}{3}-1\right)},
$$

we obtain an expression, subtracting from which the equation (9), we determine the evolutionary equation of motion for a cylindrical shell

where

$$
\left[\psi_{\tau}+\mathrm{b}_{1} \psi \psi_{\xi}+\mathrm{b}_{2} \psi_{\xi \xi \xi}+\mathrm{b}_{3} \psi_{\xi \xi}\right]_{\xi}=-\mathrm{b}_{4} \psi_{\eta \eta},
$$

$$
\begin{gathered}
\mathrm{U}_{0 \xi}=\psi \\
\mathrm{b}_{1}=\frac{1}{2 \alpha_{2}}\left[1-\frac{\alpha_{1}}{3}-\left(v-\frac{\alpha_{1}}{6}\right)^{2}\right] ; \quad \mathrm{b}_{2}=\frac{\mathrm{Rh} v_{1}}{2 \mathrm{~L}^{2} \varepsilon^{2}}\left(\frac{\alpha_{1}}{6}-v\right) \\
\mathrm{b}_{3}=\frac{\alpha_{1} \mathrm{c}_{1}}{4 \alpha_{2} \beta \varepsilon \mathrm{L}}\left[\left(\frac{\alpha_{1}}{6}-v\right)\left(\frac{2 h v_{1}}{3 \mathrm{R} \varepsilon}+\frac{1}{3}\right)+\frac{2}{3}-\frac{\mathrm{h} v_{1}}{3 \mathrm{R} \varepsilon}\right] \\
\mathrm{b}_{4}=\frac{1}{\mathrm{~A}_{2}}\left(v-\frac{\alpha_{1}}{6}\right)\left(1+\frac{\mathrm{A} \alpha_{1}}{3 \mathrm{R} \sqrt{\varepsilon}}\right)-\frac{\mathrm{LA}}{2 \mathrm{R}^{2}}\left(1-v-\frac{\alpha_{1}}{2}\right)-\frac{\mathrm{A}\left(1+v-\frac{\alpha_{1}}{3}\right.}{4 \mathrm{R} \alpha_{2} \mathrm{~A}_{2} \sqrt{\varepsilon}}
\end{gathered}
$$

\section{Results}

Increasing the reliability of pipeline structures by improving the acoustic diagnostics of microdefects in the material can be achieved by building new mathematical models that describe the wave dynamics of a cylindrical shell made of a viscoelastic material.

On the basis of a more accurate relationship between the geometric, physicalmechanical and dynamic characteristics of the shell deformation process, more stringent values of the longitudinal deformation wave velocity in the shell are determined, which make it possible to increase the accuracy of detecting invisible microdefects of the material. As a result, it is not allowed to use defective products in construction practice, i.e., the reliability of the pipelines under construction increases.

\section{Conclusion}

It was found that the effect of compensating for nonlinear dispersion properties and dissipation forms longitudinal solitary deformation waves in the shell, and their speed increases with increasing amplitude. Thus, the property of the material shell deformation process nonlinearity cannot be neglected, otherwise significant errors are inevitable arising from the use of linear models, which do not represent the possibility of detecting such an effect even at a qualitative level.

The more accurate dynamic characteristics of the wave process in a viscoelastic cylindrical shell obtained in the study make it possible to improve the acoustic methods for 
searching for invisible microdefects in a material.

Gratitude. Funding: The reported study was funded by RFBR, project number 19-010-00385 A

\section{References}

1. A.S. Volmir, Nonlinear dynamics of plates and shells (Nauka, Moscow, 1972)

2. V.V. Moskvitin, Resistance of viscoelastic materials (Nauka, Moscow, 1972)

3. U.K. Nigul, Nonlinear acoustic dynamics (Sudostroyenie, L, 1981)

4. G.V. Degtyarev, IOP Conference Series: Earth and Environmental Science 395, (2019). [EESE3951001.pdf]

5. O.V. Takhumova, V.I. Loiko, T. P. Baranovskaya, and T.I. Bondarenko IOP Conf. Series: Materials Science and Engineering 913, 042070 (2020) doi:10.1088/1757$899 X / 913 / 4 / 042070$ 